\title{
The safety and feasibility of a single incision in simultaneous resection for patients with colorectal cancer liver metastases
}

\author{
Xiao Chen ${ }^{1}$, Shida Yan $^{2}$, Hong Zhao ${ }^{1}$, Yefan Zhang ${ }^{1}$, Zhen Huang ${ }^{1}$, Jianjun Zhao ${ }^{1}$, Jianguo Zhou ${ }^{1}$, \\ Zhiyu Li ${ }^{1}$, Xinyu Bi ${ }^{1}$, Jianqiang Cai ${ }^{1}$ \\ ${ }^{1}$ Department of Hepatobiliary Surgery, National Cancer Center/Cancer Hospital, Chinese Academy of Medical Sciences and Peking Union Medical \\ College, Beijing 100021, China; ${ }^{2}$ Peking Union Medical College and Chinese Academy of Medical Science, Beijing 100730, China \\ Contributions: (I) Conception and design: X Bi, J Cai; (II) Administrative support: H Zhao, J Cai; (III) Provision of study materials or patients: Y \\ Zhang, Z Huang; (IV) Collection and assembly of data: X Chen, S Yan; (V) Data analysis and interpretation: X Chen, S Yan; (VI) Manuscript writing: \\ All authors; (VII) Final approval of manuscript: All authors. \\ Correspondence to: Jianqiang Cai; Xinyu Bi. Department of Hepatobiliary Surgery, National Cancer Center/Cancer Hospital, Chinese Academy of \\ Medical Sciences and Peking Union Medical College, Beijing 100021, China. Email: caijianqiang188@sina.com; beexy1971@163.com.
}

Background: The approach of performing a simultaneous resection for patients with colorectal cancer liver metastases has been lauded universally, but the operation procedures have differences. In general, colorectal lesions are removed laparoscopically; however, some liver metastases cannot be resected under laparoscopy. For these patients, the traditional approach of performing a simultaneous resection which utilizes the inferior abdominal midline incision and the right subcostal incision is preferred. In this study, we assessed the safety and feasibility of the single right subcostal incision approach for patients with either rectal or sigmoid colon cancer and liver metastasis who underwent simultaneous resection.

Methods: A total of 85 patients with rectal or sigmoid colon cancer and liver metastases who underwent simultaneous resection from January 2012 to December 2016 in the Cancer Hospital Chinese Academy of Medical Sciences were identified. Clinicopathological data, as well as operative and perioperative outcomes, were collected and analyzed retrospectively.

Results: Overall, 42 patients were included in this study, 26 (61.9\%) patients underwent simultaneous resection with a single surgical incision (right subcostal incision), and 16 (38.1\%) underwent simultaneous resection with dual surgical incisions (inferior abdominal midline incision and right subcostal incision). Compared to the dual-incision approach, the single-incision approach had a shorter operation time $(328.0$ vs. $420.0 \mathrm{~min}$, $\mathrm{P}=0.006$ ) but had no significant differences in total hospitalization time, postoperative hospitalization time, intraoperative blood loss, time of postoperative drainage tube extraction, time to the first postoperative bowel movement, and postoperative complications $(\mathrm{P}>0.05)$.

Conclusions: The single-incision approach (right subcostal incision) is feasible and safe for patients with either sigmoid colon or rectal cancer and liver metastases.

Keywords: Colorectal cancer; synchronous liver metastases; simultaneous resection; surgical incisions; safety; feasibility

Submitted Apr 30, 2019. Accepted for publication Aug 29, 2019.

doi: $10.21037 /$ atm.2019.09.93

View this article at: http://dx.doi.org/10.21037/atm.2019.09.93

\section{Introduction}

The liver is the most frequent hematogenous metastatic site of colorectal cancer (1-3). Currently, surgical resection remains the best treatment option for colorectal liver metastases (CRLM) (4), with markedly improved overall survival and long-term outcomes (5-7). Although the vast majority of patients with colorectal cancer and liver metastases have unresectable disease $(8,9)$, with 
the development of surgical techniques and the use of laparoscopy, there is an increasing number of studies supporting the simultaneous resection of colorectal cancer and liver metastasis as feasible and safe (10-15). Nevertheless, the surgical procedure of a simultaneous resection could still be improved, for example, the incision type.

Traditionally, due to the distance between the primary lesions and metastases, the surgical strategy for CRLM, especially for sigmoid colon or rectal cancer, requires two incision sites (an inferior abdominal midline incision and a right subcostal incision) $(16,17)$, or even a staged operation $(18,19)$. At our center, with the use of laparoscopy, we designed a novel single-incision surgical approach for simultaneous resection, which requires only a single right subcostal incision. In this prospective cohort study, the clinical data of 42 patients with sigmoid colon or rectal cancer and liver metastases who underwent simultaneous resection of both lesions at our center between January 2012 and December 2016 were collected. By comparing the patients' clinical characteristics and postoperative outcomes, we managed to compare the safety and feasibility between the single-incision and dual-incision surgical approaches and discuss the effects of the incision choice on postoperative outcomes and complications.

\section{Methods}

\section{Patient selection}

With the approval of the Institutional Review Board, 85 patients with sigmoid colon or rectal cancer and liver metastases who were treated with surgery at the Abdominal Surgery Department, Cancer Hospital of the Chinese Academy of Medical Science, Beijing, China, between January 2012 and December 2016 were included in this retrospective study. All 42 patients received an electronic colonoscope examination and underwent a biopsy, which confirmed that the primary tumors were adenocarcinoma of the sigmoid colon or rectum. Diagnosis of liver metastases was based on imaging results by hepatic magnetic resonance imaging (MRI), which was verified by two or more radiologists. Furthermore, the postoperative pathological results confirmed the diagnosis of colorectal cancer with liver metastases.

The inclusion criteria were as follows: (I) histologically confirmed colorectal adenocarcinoma liver metastases; (II) synchronous colorectal cancer liver metastases detected by preoperative imaging or during operation; (III) the primary tumor and all liver metastases could be resected curatively according to preoperative imaging assessment; and (IV) no extrahepatic metastases.

The exclusion criteria were as follows: (I) temporary or permanent ostomy; (II) colorectal lesions that cannot be removed by laparoscopic operation; (III) inability to tolerate the pneumoperitoneum; (IV) history of laparoscopic hepatectomy for liver metastases; or (V) liver metastases that can be radically resected by laparoscopic hepatectomy.

The indications of a laparoscopic hepatectomy include a solitary lesion located in the peripheral segments of the liver and not adjacent to major vasculature, and multiple lesions that can be resected with a single anatomic hepatectomy with a clear margin. The indications of open hepatectomy include a solitary lesion adjacent to major vasculature that prevents negative margin or safe manipulation, a large solitary lesion that cannot obtain margin-free resection or safe manipulation, and multiple liver metastases with bilobar distribution and complicated manipulation. Whether it is laparoscopic or open hepatectomy, we need to reserve an adequate volume of future liver remnant for a successful recovery after surgery.

\section{Surgical treatment}

The choice of a single-incision versus a dual-incision approach is dependent on the surgeon's preference and patient's intent. The laparoscopic process was consistent in the two groups. Under general anesthesia, the patient was placed in the Tren-delenburg position. A $10-\mathrm{mm}$ observation trocar was inserted below the umbilicus, and then three ports were placed after the establishment of pneumoperitoneum. One 12-mm port was placed in the right lower quadrant, one $5-\mathrm{mm}$ port was inserted in the right upper abdomen, and a further 5 -mm port was placed in the left lower abdomen. In the single-incision group (Figure 1), the operation was performed with the assistance of laparoscopy at the beginning, which includes disconnection of relevant arteries and veins of the intestinal segments to be removed, mesentery isolation, intestine baring, and disconnection of the intestinal segment 3 to 5 centimeters distal to the tumor. Then, disconnection of the intestinal segment proximal to the primary lesion and subsequent intestinal tract reconstruction would be performed by laparotomy with the right oblique subcostal incision. In the end, liver metastasis resection would also be accomplished from the same incision site. In the dualincision group (Figure 2), the laparoscopic part is the same 


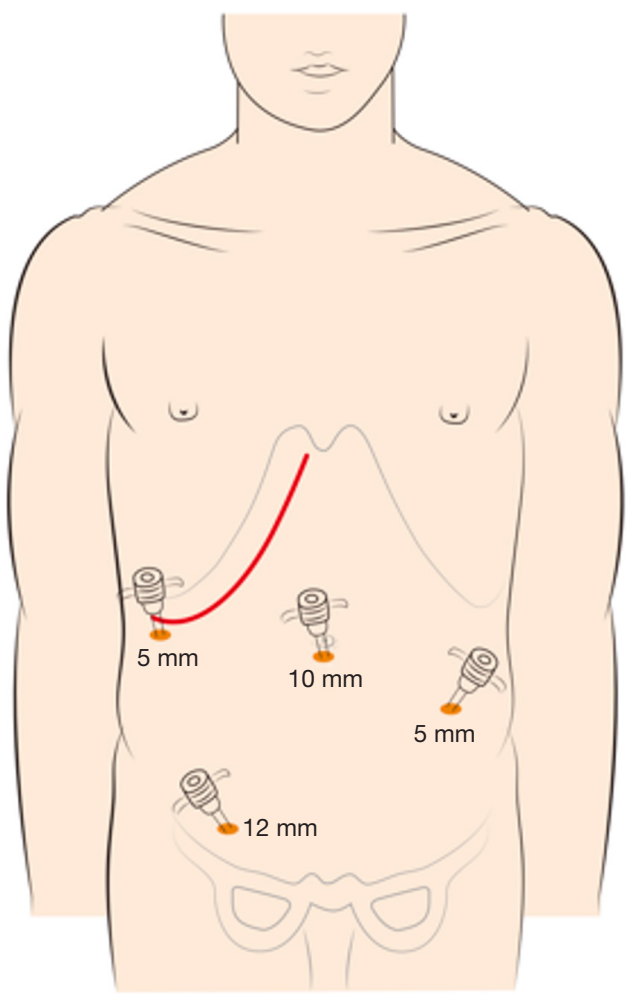

Figure 1 The mode of single-incision. Firstly, we disconnected the relevant arteries and veins of the intestinal segments to be removed, isolated mesentery, bared intestine and disconnected the intestinal segment three to five centimeters distal to the tumor with the assistant of laparoscopy (orange cut). And then the disconnection of the intestinal segment proximal to the primary lesion, intestinal tract reconstruction and liver metastases resection were performed by laparotomy with the right oblique subcostal incision (red line).

as that in the single-incision group. Then, disconnection of the intestinal segment proximal to the primary lesion and intestinal tract reconstruction would be achieved by laparotomy through the midline incision of the inferior abdomen. Finally, the right oblique subcostal incision would be performed to finish the liver metastasis resection.

\section{Outcome evaluation and statistical analysis}

For each patient, the intraoperative and postoperative outcomes were evaluated, including operation time, total hospital stay, postoperative hospital stay, intraoperative blood loss, time of removal of drainage tubes, and postoperative complications. Surgical outcomes between

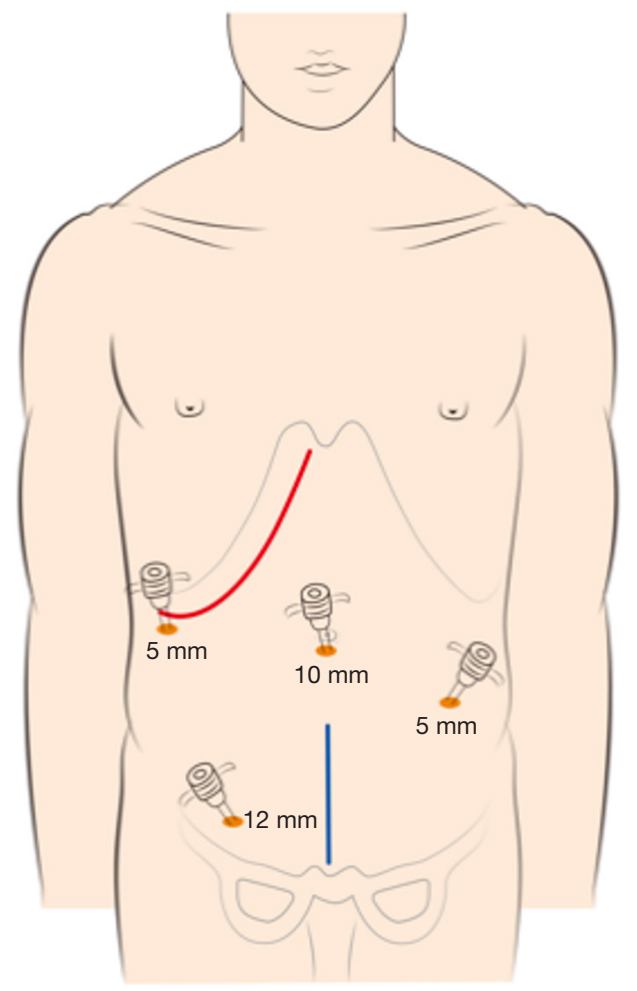

Figure 2 The mode of dual-incision. Firstly, we disconnected the relevant arteries and veins of the intestinal segments to be removed, isolated mesentery, bared intestine and disconnected the intestinal segment three to five centimeters distal to the tumor with the assistant of laparoscopy (orange cut). Secondly, the disconnection of the intestinal segment at the proximal of the primary lesion and intestinal tract reconstruction were achieved by laparotomy through the midline incision of the inferior abdomen (blue line). Finally, liver metastases resection was performed through the right oblique subcostal incision (red line).

the single-incision group and dual-incision group were compared using the $\chi^{2}$ test for enumeration data and the Mann-Whitney $U$ test for measurement data. Statistical significance was defined as $\mathrm{P}<0.05$. All of the analyses were performed using the statistical software SPSS 21.0 (SPSS Inc., Chicago, IL, USA).

\section{Results}

\section{Patient characteristics}

Among the 85 patients, 21 patients with liver metastases removed radically by laparoscopic hepatectomy were excluded, 9 patients with rectal cancer liver metastases 
who underwent laparoscopic abdominoperineal resection or laparoscopic low anterior resection combined with preventive ileostomy were excluded, 7 patients who underwent open radical resection for rectal cancer were excluded, and 6 patients converting to laparotomy in laparoscopic radical resection for rectal cancer were excluded.

Overall, 42 patients were included in this study. The demographic and clinical characteristics of the patients are summarized in Table 1. Among all patients, 28 (66.7\%) were male, and 14 (33.3\%) were female, while the age range was 34-74, and the median age was 57.5. The single-incision group had $26(61.9 \%)$ patients, and the dual-incision group had $16(38.1 \%)$ patients.

In this study, $18(42.3 \%)$ patients had cancer that primarily originated from the sigmoid colon, and the singleincision group had a larger number of patients with primary colon cancer than did the dual-incision group, but there was no noticeable difference $(\mathrm{P}>0.05)$. There was no significant difference in the condition of local tumor node (TN) stage between the two groups $(\mathrm{P}>0.05)$.

The median number of liver metastases was 3.0 and 3.0 , with a median size of CRLM of 2.8 and $2.0 \mathrm{~cm}$ in the single-incision group and the dual-incision group, respectively $(\mathrm{P}>0.05)$. We used Couinaud' classification for the definition of liver segments. Major hepatectomy was defined as resection of more than two liver segments. The extent of liver resection and the distribution of liver metastases were comparable between the two groups $(\mathrm{P}>0.05)$.

Among the 42 patients included in this study, 24 patients were diagnosed with rectal cancer. Eleven patients were diagnosed with tumors 6 to $10 \mathrm{~cm}$ from the anus, and 13 patients were diagnosed with tumors 10 to $15 \mathrm{~cm}$ from the anus through colonoscopy combined with rectal touch. For patients with rectal cancer liver metastases, 13 patients received neoadjuvant chemotherapy, 5 patients received neoadjuvant chemotherapy combined with targeted therapy, 3 patients received neoadjuvant concurrent chemoradiotherapy, and 3 patients received no neoadjuvant therapy before operation.

All the patients underwent laparoscopic total mesorectal excision for rectal or sigmoid colon cancer. The total number of lymph nodes harvested from the 42 patients included in this study ranged from 4 to 53 , with a median number of 19.5. In the single-incision group, N0, N1, and $\mathrm{N} 2$ accounted for $23.1 \%, 46.2 \%$, and $30.7 \%$ of cases, respectively. In the dual-incision group, N0, N1, and $\mathrm{N} 2$ accounted for $6.3 \%, 56.3 \%$, and $37.4 \%$ of cases, respectively. According to postoperative pathology, all the rectal or sigmoid colon cancers were resected with negative margins $(\geq 2 \mathrm{~cm})$, and liver metastases were removed with clear margins ( $\mathrm{R} 0$ resection).

\section{Intra- and postoperative outcomes}

Twenty-six patients underwent single-incision surgery, while the other 16 underwent dual-incision surgery. There was no significant difference between the two groups regarding total hospital stay, postoperative hospital stay, volume of intraoperative blood loss, time of postoperative drainage tube extraction (including gastric tube, urinary catheter, pelvic and peritoneal cavity drainage tube), and the time to the first postoperative bowel movement $(\mathrm{P}>0.05)$ (Table 2). However, concerning the operation time, the duration was shorter in the single-incision group than in the dual-incision group (328.0 vs. 420.0 min, respectively; $\mathrm{P}=0.006$ ).

Concerning the postoperative complications, there was still no significant difference between the two groups. Among the 42 patients, there was no perioperative death or postoperative hemorrhage. The postoperative infection rate was lower in the single-incision group $(26.9 \%)$ than in the dual-incision group (31.3\%), but no significant difference was observed $(\mathrm{P}>0.05)$. Among the 6 patients in the single-incision group with postoperative infection, 5 suffered from abdominal infection, and 1 patient had a pulmonary infection. In the dual-incision group, 5 patients were diagnosed with an infection, including 3 patients with an abdominal infection, 1 patient with pulmonary infection, and 1 patient with a urinary tract infection. After proper anti-infection treatment, the infections in all affected patients were controlled effectively. Meanwhile, there was 1 case of anastomotic leakage in the single-incision group, which occurred on the 6th day after surgery, and the patient recovered by undergoing temporary double-lumen ileostomy. Additionally, there was no significant difference between the two groups regarding anastomotic leakage $(\mathrm{P}>0.05)$.

\section{Discussion}

Although the surgical strategies for colorectal cancer liver metastases are debated, an increasing number of studies has demonstrated that compared to a staged resection, a simultaneous resection can obviate a second operation, shorten operation time, reduce intraoperative blood loss, shorten postoperative hospital stay, and reduce hospital 
Table 1 Patient characteristics

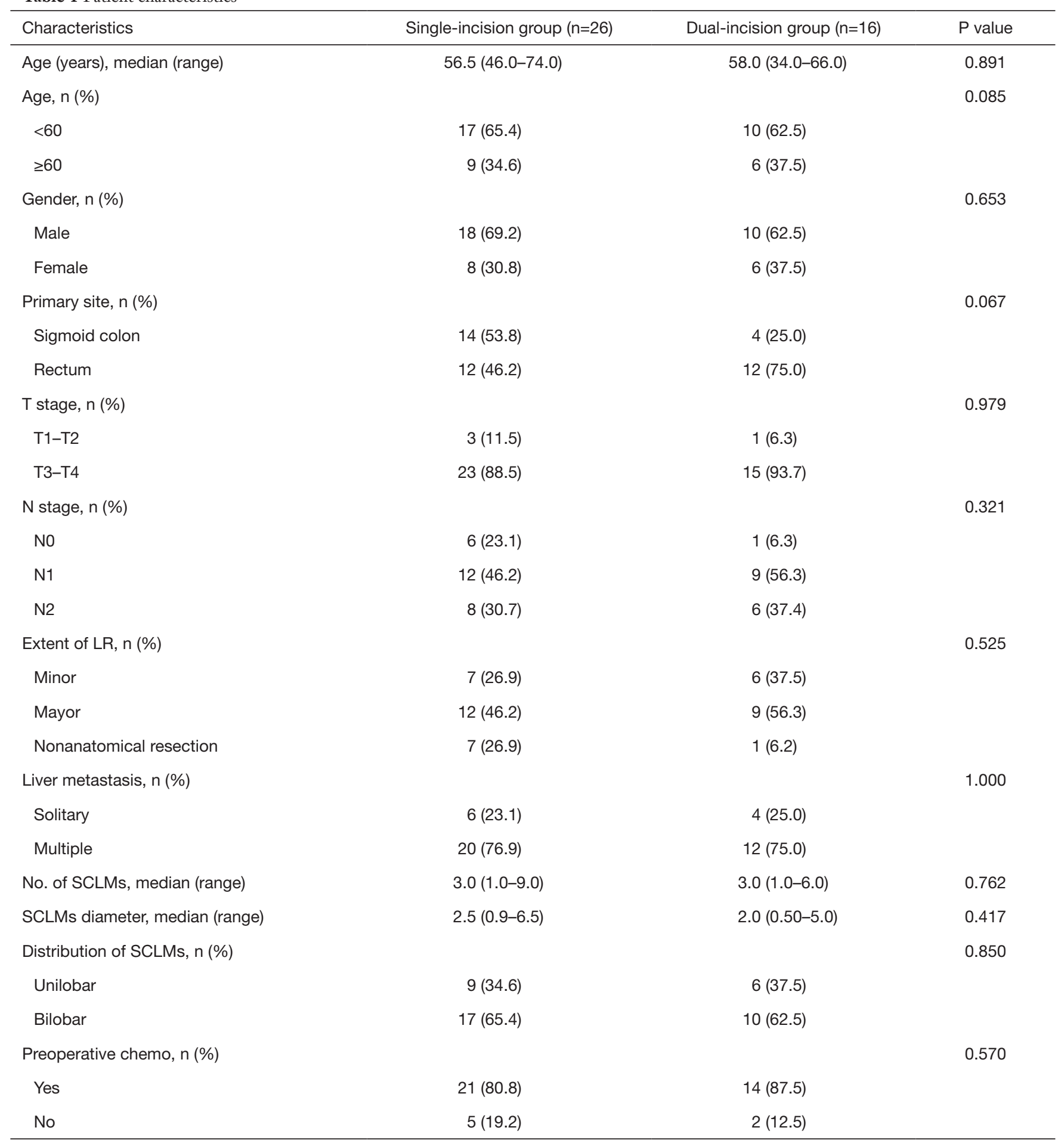

LR, liver resection; SCLMs, synchronous colorectal liver metastases; chemo, chemotherapy. 
Table 2 Comparative analysis of intra- and postoperative outcomes

\begin{tabular}{|c|c|c|c|}
\hline Variable & Single-incision group $(\mathrm{n}=26)$ & Dual-incision group $(n=16)$ & $P$ value \\
\hline Postoperative hospital stay (days), median (range) & $10.0(8.0-36.0)$ & $10.0(8.0-20.0)$ & 0.620 \\
\hline Operation time (min), median (range) & $328.0(245.0-570.0)$ & $420.0(245.0-760.0)$ & 0.006 \\
\hline Intra-operative blood loss (mL), median (range) & $300.0(100.0-1,200.0)$ & $250.0(50.0-900.0)$ & 0.316 \\
\hline Gastric tube & $4.5(1.0-8.0)$ & $5.0(3.0-14.0)$ & 0.413 \\
\hline Urinary catheter & $6.0(2.0-21.0)$ & $7.0(3.0-17.0)$ & 0.454 \\
\hline Pelvic cavity drainage tube & $8.0(6.0-21.0)$ & $8.0(4.0-14.0)$ & 0.662 \\
\hline \multicolumn{4}{|l|}{ Postoperative complication (n,\%) } \\
\hline Infection & $6(23.1)$ & $5(31.3)$ & 0.823 \\
\hline Abdominal infection & $5(19.2)$ & $3(18.8)$ & 1.000 \\
\hline Pulmonary infection & $1(3.8)$ & $1(6.3)$ & 1.000 \\
\hline Urinary tract infection & 0 & $1(6.3)$ & 0.804 \\
\hline Anastomotic leakage & $1(3.8)$ & 0 & 1.000 \\
\hline Hemorrhage & 0 & 0 & - \\
\hline
\end{tabular}

costs. Also, there is no significant difference between the two surgical strategies regarding short-term and long-term outcomes $(10,11,20,21)$. Our experiences from the clinical practice at our center attest to safety and efficacy of treating CRLM patients using simultaneous surgical resection $(13,14)$.

Moreover, the ideal surgical procedure and incision approach for the simultaneous resection of CRLM, in which various types of incisions have been applied, is still controversial. When choosing an ideal surgical incision approach, the following principles should be considered: providing adequate exposure, minimally interfering with abdominal wall anatomy and function, rapidly entering the peritoneal cavity, and fulfilling a cosmetic result. The midline and paramedian incisions are the most common incision approaches applied in colorectal surgery and entail less blood loss and no muscle damage or nerve injury. However, the right subcostal incision is also used in colorectal surgery (22). In liver surgery, the Kocher subcostal incision, i.e., the right subcostal oblique incision, is often used, which has the advantages of hepatic mobilization and vascular control. Considering the needs of both colorectal and liver resection, the extended midline incision and lower midline plus right subcostal incision were the most commonly used, while other types of incision approaches have also been innovated. For instance, Hsu et al. (23) report a type of reversed L-shaped incision that was used for right hemicolectomy combined with liver resection. However, with regards to simultaneous the resection of sigmoid colon or rectal cancer with synchronous liver metastases, there were few improvements in the incision approach.

At our center, for patients with sigmoid colon or rectal cancer and CRLM and for whom removing liver metastases was infeasible, we used a single-incision approach, which was the right subcostal oblique incision. Our study included 42 patients with sigmoid colon or rectal cancer and CRLM, of whom 26 underwent a singleincision approach, and 16 underwent a dual-incision approach. We compared hospitalization time, postoperative hospitalization time, operation time, intraoperative blood loss, time of postoperative drainage tube extraction, time to the first postoperative bowel movement, and postoperative complications between the two groups, and the only 
significant difference was the operation time. The median duration of operating time in the single-incision and dualincision groups was 328.0 and $420.0 \mathrm{~min}$, respectively $(\mathrm{P}=0.006)$. In dual-incision group, after radical resection of primary lesions and intestinal tract reconstruction by laparoscopic approach and laparotomy thorough the midline incision of the inferior abdomen, we re-disinfected and re-laid sterile sheets for patients before liver metastases resection. Therefore, the process of re-disinfection and relaying sterile sheets, and the additional midline incision of the inferior abdomen and its closure increased the operation time. Few postoperative complications occurred in either group. Postoperative infection is the most worrisome issue in simultaneous resection due to intestinal lumen exposure. The rate of infection in both groups was similar $(26.9 \% \mathrm{vs}$. $31.3 \%, \mathrm{P}>0.05)$. The only instance of anastomotic leakage occurred in the single-incision group, and there was no difference in the rate of anastomotic leakage between the two groups.

Regarding the single-incision operative approach, the most significant concern is the ability to remove sigmoid or rectal lesions successfully. During the single-incision operation, we first disconnected the relevant arteries and veins, isolated the mesentery, stripped the intestine, and disconnected the intestinal segment distal to the lesion with the assistance of laparoscopy. Therefore, only the disconnection of the intestinal segment proximal to the primary lesion and intestinal tract reconstruction needed to be performed with an open approach. In our experience, the latter steps were feasible as long as the mesentery isolation was adequate. With the development of the laparoscopic technique, several studies have suggested that the laparoscopic approach is effective and safe for simultaneous resection of colorectal cancer and CRLM in appropriate patients $(12,24-27)$. For patients unable to undergo laparoscopic operations, such as the patients in our study, the single-incision open approach is available with the benefit of shortening the operation time shortening and reducing both abdominal wall trauma and pain.

This study has several limitations. The patient numbers of both groups were relatively limited, and the patients were identified from a single center. Due to the availability of laparoscopic surgery, fewer patients in our hospital received an open approach. To ensure the value of this study, we chose relatively strict inclusion criteria. Furthermore, due to the limitation of time, we were unable to analyze the long-term outcomes for both incision approaches, including overall survival, disease-free survival, and long-term complications, which require further research.

\section{Conclusions}

We presented the retrospective outcomes of simultaneous resections of either sigmoid colon or rectal cancer and liver metastases utilizing a single right subcostal incision or lower midline plus right subcostal incisions conducted in our hospital. Our results revealed that the singleincision approach had a shorter operation time and had no significant differences with the dual-incision approach with respect to postoperative recovery and complications. Thus, the results support the conclusion that the single-incision approach (right subcostal incision) is feasible and safe for patients with sigmoid colon or rectal cancer and liver metastases. Furthermore, the long-term outcomes of this single-incision approach should be studied in a prospective multicenter randomized controlled trial, and continued improvements of the incision technique should continue.

\section{Acknowledgments}

Funding: This study was supported by State Key Project on Infection Diseases of China (Grant No. 2017ZX10201021007-003), Capital health research and development of special (Grant No. 2018-1-4021), National Natural Science Foundation of China (Grant No. 81672461), CAMS Innovation Fund for Medical Sciences (CIFMS) (Grant No. 2016-I2M-1-001, Grant No. 2017-12M-4-002).

\section{Footnote}

Conflicts of Interest: The authors have no conflicts of interest to declare.

Ethical Statement: The authors are accountable for all aspects of the work in ensuring that questions related to the accuracy or integrity of any part of the work are appropriately investigated and resolved. The study is approved by the Institutional Review Board.

\section{References}

1. McMillan DC, McArdle CS. Epidemiology of colorectal liver metastases. Surg Oncol 2007;16:3-5.

2. Donadon M, Ribero D, Morris-Stiff G, et al. New Paradigm in the Management of Liver-Only Metastases From Colorectal Cancer. Gastrointestinal Cancer 
Research: GCR 2007;1:20-7.

3. Wu JB. Transcatheter arterial chemoembolization for liver metastases. Electronic Journal of Liver Tumor 2018;5:30-3.

4. Kemeny MM. Surgery should be the primary treatment of synchronous colorectal metastases in the asymptomatic patient. Ann Surg Oncol 2006;13:140-1.

5. Fong Y, Cohen AM, Fortner JG, et al. Liver resection for colorectal metastases. J Clin Oncol 1997;15:938-46.

6. Beard SM, Holmes M, Price C, et al. Hepatic Resection for Colorectal Liver Metastases: A Cost-Effectiveness Analysis. Ann Surg 2000;232:763-76.

7. Robertson DJ, Stukel TA, Gottlieb DJ, et al. Survival after hepatic resection of colorectal cancer metastases: a national experience. Cancer 2009;115:752-9.

8. Abdalla EK, Vauthey JN, Ellis LM, et al. Recurrence and Outcomes Following Hepatic Resection, Radiofrequency Ablation, and Combined Resection/Ablation for Colorectal Liver Metastases. Ann Surg 2004;239:818-25.

9. Dexiang Z, Li R, Ye W, et al. Outcome of Patients with Colorectal Liver Metastasis: Analysis of 1,613 Consecutive Cases. Ann Surg Oncol 2012;19:2860-8.

10. Reddy SK, Pawlik TM, Zorzi D, et al. Simultaneous resections of colorectal cancer and synchronous liver metastases: a multi-institutional analysis. Ann Surg Oncol 2007;14:3481-91.

11. Martin RCG, Augenstein V, Reuter NP, et al. Simultaneous Versus Staged Resection for Synchronous Colorectal Cancer Liver Metastases. J Am Coll Surg 2009;208:842-50.

12. Ferretti S, Tranchart H, Buell JF, et al. Laparoscopic Simultaneous Resection of Colorectal Primary Tumor and Liver Metastases: Results of a Multicenter International Study. World J Surg 2015;39:2052-60.

13. Zhang YF, Mao R, Chen X, et al. Prognostic Analysis of 102 Patients with Synchronous Colorectal Cancer and Liver Metastases Treated with Simultaneous Resection. Chin Med J (Engl) 2017;130:1283-9.

14. Li Y, Bi X, Zhao J, et al. Simultaneous hepatic resection benefits patients with synchronous colorectal cancer liver metastases. Chin J Cancer Res 2016;28:528-35.

15. Mayo SC, Pulitano C, Marques H, et al. Surgical management of patients with synchronous colorectal liver metastasis: a multicenter international analysis. J Am Coll Surg 2013;216:707-16; discussion 716-8.

16. Moug SJ, Horgan PG. The role of synchronous procedures in the treatment of colorectal liver metastases.
Surg Oncol 2007;16:53-8.

17. Bretagnol F, Hatwell C, Farges O, et al. Benefit of laparoscopy for rectal resection in patients operated simultaneously for synchronous liver metastases: preliminary experience. Surgery 2008;144:436-41.

18. Chow FC, Chok KS. Colorectal liver metastases: An update on multidisciplinary approach. World J Hepatol 2019;11:150-72.

19. Chedid AD, Villwock Mde M, et al. Prognostic factors followingliver resection for hepatic metastases from colorectal cancer. Arq Gastroenterol 2003;40:159-65.

20. Abelson JS, Michelassi F, Sun T, et al. Simultaneous Resection for Synchronous Colorectal Liver Metastasis: the New Standard of Care? J Gastrointest Surg 2017;21:975-82.

21. Silberhumer GR, Paty PB, Temple LK, et al. Simultaneous resection for rectal cancer with synchronous liver metastasis is a safe procedure. Am J Surg 2015;209:935-42.

22. Theodosopoulos T, Yiallourou AI, Dafnios N, et al. Right Kocher's incision: a feasible and effective incision for right hemicolectomy: a retrospective study. World J Surg Oncol 2012;10:101.

23. Hsu KF, Yu JC, Chen TW, et al. Experience with reversed L-shaped incision for right hemicolectomy combined with liver resection. World J Surg 2009;33:2679-82.

24. Gorgun E, Yazici P, Onder A, et al. Laparoscopic versus open 1-stage resection of synchronous liver metastases and primary colorectal cancer. Gland Surg 2017;6:324-9.

25. Xu X, Guo Y, Chen G, et al. Laparoscopic resections of colorectal cancer and synchronous liver metastases: a case controlled study. Minim Invasive Ther Allied Technol 2018;27:209-16.

26. Kim SH, Lim SB, Ha YH, et al. Laparoscopic-assisted combined colon and liver resection for primary colorectal cancer with synchronous liver metastases: initial experience. World J Surg 2008;32:2701-6.

27. Ratti F, Catena M, Di Palo S, et al. Laparoscopic Approach for Primary Colorectal Cancer Improves Outcome of Patients Undergoing Combined Open Hepatic Resection for Liver Metastases. World J Surg 2015;39:2573-82.

Cite this article as: Chen X, Yan S, Zhao H, Zhang Y, Huang Z, Zhao J, Zhou J, Li Z, Bi X, Cai J. The safety and feasibility of a single incision in simultaneous resection for patients with colorectal cancer liver metastases. Ann Transl Med 2019;7(20):547. doi: $10.21037 /$ atm.2019.09.93 